\title{
Factors affecting providers' delivery of intermittent preventive treatment for malaria in pregnancy: a five-country analysis of national service provision assessment surveys
}

\author{
Mathieu Maheu-Giroux and Marcia C Castro*
}

\begin{abstract}
Background: Intermittent preventive treatment in pregnancy (IPTp) delivered during antenatal care (ANC) visits has been shown to be a highly efficacious and cost-effective intervention. Given the high rates of ANC attendance in sub-Saharan Africa, the current low IPTp coverage represents considerable missed opportunities. The objective of this study was to explore factors affecting provider's delivery of IPTp during ANC consultations.

Methods: Data from five nationally representative service provision assessment surveys informed the statistical analyses (Kenya, Namibia, Rwanda, Tanzania, and Uganda; 2006-2010). Poisson regression models with robust/ clustered standard errors were used to estimate the effect of different determinants on IPTp delivery from 4,971 observed ANC consultations.
\end{abstract}

Results: The five major modifiable determinants of IPTp delivery were: 1 ) user-fees for ANC medicines (relative risk $(\mathrm{RR})=0.76 ; 95 \%$ confidence intervals (95\% Cl): 0.62-0.93); 2) facilities having IPTp guidelines (RR $=1.12 ; 95 \% \mathrm{Cl}$ :

1.01-1.24); 3) facilities having implemented IPTp as part of their routine ANC services offering (RR $=4.18$; $95 \% \mathrm{Cl}$ : $1.75-10.01)$; 4) stock-outs of sulphadoxine-pyrimethamine $(\mathrm{RR}=0.40 ; 95 \% \mathrm{Cl}: 0.27-0.60)$; and, 5) providers having received IPTp training ( $R R=1.21 ; 95 \% \mathrm{Cl}: 1.09-1.35)$. Using the population-attributable fraction, it was estimated that addressing these barriers jointly could lead to a $31 \%$ increase in delivery of this intervention during ANC consultations. Of these four potentially modifiable determinants, training of providers for IPTp had the largest potential impact.

Conclusions: If proved to be cost-effective, dispensing IPTp training to ANC providers should be prioritized. Multifaceted approaches targeted in areas of low coverage and/or type of facilities least likely to provide this intervention should be implemented if the Roll Back Malaria target of 100\% IPTp coverage by 2015 is to be attained.

\section{Background}

Pregnant women are especially vulnerable to malaria as parasitaemia during pregnancy can lead to serious adverse maternal, foetal, and infant health outcomes [1]. In sub-Saharan Africa, where malaria burden is concentrated, there was an estimated 30 million pregnancies at risk of this parasitic disease in 2007 [2]. The World Health Organization (WHO) recommends a three-pronged approach to address this major public health issue: 1) prompt and effective case management of clinical malaria

\footnotetext{
* Correspondence: mcastro@hsph.harvard.edu

Department of Global Health \& Population, Harvard School of Public Health, 665 Huntington Avenue, Bldg I, Room 1113, Boston, MA 02115, USA
}

and anaemia; 2) distribution of insecticide-treated bed nets; and, 3) intermittent preventive treatment in pregnancy (IPTp) with sulphadoxine-pyrimethamine (SP) [3]. IPTp consists of presumptive provision of anti-malarials to pregnant women, shortly after quickening and at intervals of at least four weeks, under the direct observation of health workers. This intervention has been shown to be safe and highly effective to prevent maternal anaemia, low birth weight, and neonatal mortality [4-7], even in areas of recorded resistance to SP [8]. Further, IPTp delivered through antenatal care clinics (ANC) is considered very cost-effective with an incremental cost-effectiveness ratio of \$1.02 (2007 USD) per disability-adjusted life-year averted [9]. 
Despite the fact that $75 \%$ of pregnant women in subSaharan Africa visit ANC services at least twice during their pregnancy, the proportion of women receiving at least two SP doses remained stubbornly low at $22 \%$ in 2009-2011 [10,11]. Such missed opportunities to deliver IPTp through ANC have been described as a 'failure of the public health community' [12]. Further, the actual coverage levels of IPTp are disconcerting given the Roll Back Malaria Partnership goal of $80 \%$ IPTp coverage with at least two SP doses by 2010 (and 100\% by 2015) [13] and the President's Malaria Initiative's target of $85 \%$ coverage [14].

A systematic review of qualitative studies that explored barriers to IPTp coverage showed that unclear policy guidance, drug stock-outs, user fees, poor organization at health facility, underperformance of healthcare providers, and low ANC attendance were effectively impeding delivery of this intervention [12]. Other authors have also suggested that confusion among healthcare providers, related to the previous WHO guidelines regarding timing and number of IPTp doses, was one of the key factors influencing coverage of this intervention $[11,15]$. These recommendations were recently revised in an attempt to provide clearer guidance and WHO now advocates that SP should be provided at each of the four scheduled antenatal care visits [16]. A recent metaanalysis found that the number and timing of ANC visits, parity, education level, socio-economic status, knowledge about malaria and IPTp, and use of ITN were key determinants of IPTp uptake at the individual level [12]. Few interventions have so far been proposed to increase coverage of IPTp. Community-based distribution could effectively complement ANC-based delivery of IPTp $[17,18]$ but there is some evidence that this could concurrently reduce women's ANC attendance [19,20]. Potential alternatives were principally aimed at promoting IPTp in the community, increasing ANC coverage, and improving providers' performance [21-23].

In light of the important gap between ANC attendance and IPTp uptake, it is hypothesized that interventions aimed at health providers are likely to be most costeffective. A multi-country quantitative analysis of factors affecting providers' delivery of IPTp has yet to be conducted, however. The aim of this study is to investigate providers' determinants of IPTp delivery in sub-Saharan Africa. Such information could provide crucial evidence to effectively design and implement interventions leading to significant increases in IPTp coverage in malariaendemic areas. To this end, service provision assessment (SPA) surveys, conducted in five countries where a national IPTp policy was implemented at the time of the survey (Kenya, Namibia, Rwanda, Tanzania, Uganda), were used. By comprehensively assessing the preparedness of health facilities to provide maternal care and collecting detailed and standardized information on the performance of IPTp delivery systems, these nationally representative surveys provide a unique opportunity to explore supplyside determinants of IPTp delivery.

\section{Methods}

SPA surveys have been conducted by The DHS Program since 1999 in 11 countries so far. Standardized methodology and instruments are used to provide comparable information on key indicators of formal sector health services related to child health, maternal and newborn health, family planning, selected infectious diseases services, basic surgery, and non-communicable diseases. As such, pharmacies and individual doctor's practices are not usually included in these surveys. SPA surveys consist of four main questionnaires: 1) an inventory questionnaire that collects information on availability of different services and general service readiness at the health facility; 2) a health worker interview questionnaire; 3 ) an observation protocol of client-provider consultations for selected services; and, 4) an exit interview questionnaire for clients of these selected services.

\section{Inclusion criteria}

Only recent surveys conducted after 2000, that implemented the observation protocol for ANC client-provider consultations, and that were performed at a time when the country had implemented a national IPTp policy were included. For this reason the SPAs from Namibia and Rwanda are included in this study even though they abandoned their national IPTp policy in 2010 and 2008, respectively, following important declines in malaria transmission experienced by these two countries. Hence, data on delivery of IPTp and its determinants came from five SPA surveys conducted between 2006 and 2010 in Kenya (2010) [24], Namibia (2009) [25], Rwanda (2007) [26], Tanzania (2006) [27], and Uganda (2007) [28]. The Kenya 2004 SPA [29] was not included because interviewers did not record whether anti-malarial prophylaxis was observed to be administered as directly observed therapy (DOT) or only prescribed to the ANC clients.

\section{Survey design}

The sampling for each SPA was designed to allow for indicators to be representative at the national and regional levels, by type of facility and by managing authority. The sample of facilities selected for inclusion was obtained from a master list of all health facilities. National referral hospitals and regional general hospitals are often oversampled, as well as facilities providing specific services. Survey weights are assigned to each facility to correct for such differential sampling. Three of the included SPA surveys used this design. In contrast, Namibia performed a census of all its health facilities, and Rwanda included all 
of its government-owned establishments, all private facilities with at least five employees, and one third of private facilities with three or four employees.

Providers of health services were sampled among those who were present in the facility on the day of the survey and who provided the services being assessed by the SPA survey. The target was to interview an average of eight providers by facility, including all those whose consultations were observed. Sampling weights were constructed in order to account for differential sampling of providers with distinct qualifications in a facility type and region. The sample of health providers was generally considered representative of the staff who provides the services being assessed, except in the very few instances when a special training event or evaluation for a group of workers would have taken them away from their post on the day of the survey.

It is generally difficult to obtain a sampling frame of eligible clients that would attend the assessed services on any particular day. Therefore, the SPA surveys used a convenience sample of clients for the observation protocols and exit interviews. Specifically, clients were selected as they arrived for consultations at the facility. When multiple eligible clients were available, interviewers selected two new clients for every follow-up case. A target of five clients was included, with a maximum of 15 observations in any given facility for each assessed service. Exit interviews were attempted for every client observed during a consultation. All SPA surveys used this sampling design except the 2010 Kenyan SPA. For this latter survey, clients entering the facility were systematically sampled until a maximum of five observations per provider were obtained and no more than 15 observations in any given facility for each service. Sampling weights used the facility weights described above and adjusted for over-representation of observations based on the compiled total number of clients of each service of interest seen on the day of the survey (note that facility, providers and clients' sampling weights are not provided in the Rwanda 2007 SPA). In a few instances, the sample of clients present on the day of the survey might not be representative of clients normally receiving health services if the survey coincided with special events such as a health fair or campaign.

\section{Data processing}

Databases with observations of ANC consultations and exit interviews were linked to the provider's interview and facility questionnaires using their unique identifiers. Although the survey instruments described above were standardized, country differences in the classification of the different facility types and of a provider's qualifications exist. Hence, the classification of these variables was harmonized according to the criteria described in
Additional file 1 before merging the five SPAs together. The variables considered in this study were:

Facility level: 1) facility type; 2) managing authority (public vs private); 3) whether the facility charged userfees for medicines given during ANC consultations (yes/ no, as reported by the manager of ANC services); 4) whether the facility had guidelines or protocol for IPTp; 5) whether the manager of ANC services claimed that IPTp was routinely offered to antenatal clients; and, 6) whether the facility had tablets of SP (Fansidar, Metakelfin, Orodar) available in its inventory on the day of the survey (stockouts are defined as not having SP in the inventory on the day of the survey). Information on these variables was extracted from the facility questionnaire.

Provider level: 1) professional/technical/medical qualification of the provider; 2) whether the provider reported to have received supervision or technical support from a supervisor in the facility or outside of the facility in the previous six months; and, 3) whether the provider had received any pre-service or in-service training for IPTp in the preceding year. Information on these variables was extracted from the providers' questionnaire.

Client level: 1) whether the provider administered antimalarial prophylaxis as DOT (as observed by the interviewer); 2) primigravidae status; 3) whether it was the first ANC visit at the facility for the current pregnancy; 4) education level; 5) age; and, 6) length of pregnancy (weeks). Information on these variables was extracted from the observation protocol of client-provider consultations and the clients' exit interview questionnaire.

Namibia's IPTp policy only targeted its malaria-endemic areas and observations of consultations outside these areas were therefore excluded. Similarly, the policy in place in Kenya during the 2010 SPA specifically targeted three provinces where malaria was most endemic (Nyanza, Coast, and Western) [24] but the decision to include observations from all provinces was made because IPTp delivery did not differ between the target and non-targeted provinces (i.e., 44 vs 42\%). Finally, because IPTp administration should be avoided in the first trimester (i.e., before quickening), only consultations from clients that were 16 weeks pregnant or more were included.

\section{Statistical analyses}

The main outcome is whether the provider was observed to provide anti-malarial prophylaxis as DOT during an ANC consultation (hereinafter referred to as IPTp). The frequency of this outcome was high and reporting odds ratio will overstate the relative risk (RR) association the quantity of interest for public health research. LogPoisson models provide consistent estimates of RR but, in case of common binary outcomes, Poisson errors will overestimate binomial errors if a robust error variance is 
not used [30]. Hence, a modified Poisson regression model that used generalized estimating equation to perform the unbiased variance estimation, taking into account clustering of observations within providers and facilities [31,32], was adopted. This model takes the following form:

$$
\begin{gathered}
\mathrm{Y}_{i j k} \sim \text { Poisson }\left(\pi_{i j k}\right) \\
\log \left(\pi_{i j k}\right)=\alpha+\beta\left(\text { Facility }_{k}\right)+\delta\left(\text { Provider }_{j}\right)+\gamma\left(\text { Individual }_{i}\right) \\
+\omega\left(\text { Country }_{k}\right)+\varepsilon_{i j k}
\end{gathered}
$$

where $\pi_{i j k}$ is the probability that women $i$ is being given anti-malarial prophylaxis by provider $j$ in facility $k$; $\alpha$ is the intercept; $\beta$ is a vector of coefficients for facility-level variables; $\delta$ is a vector of coefficients for provider-level variables; $\gamma$ is a vector of coefficients for the individual women's variables; and $\omega$ is the vector of coefficients for country fixed effects. Robust standard errors were obtained using an exchangeable correlation structure at the facility level. Because observations of client-provider's consultation are perfectly nested within facilities, clustering the standard errors at this upper level through the sandwich variance estimator will also take into account clustering of consultations at the provider level [33]. Following the rationale of Solon et al. [34], who discussed three situations where using sampling weights is justified (none of which applied to the present case), sampling weights were not used in the regression analyses.

Exploratory data analyses suggested that the relationship between the outcome and week of pregnancy was not linear. The functional form of this variable was hence modelled using a cubic b-spline with five degrees of freedom. Observations with missing values for the outcome were excluded from the analysis and covariates with missing observations were retained in the analysis using the missing indicator method [35]. All variables were first entered in univariate models before the full multivariate model was fit. Pooling data from these five countries was deemed appropriate as key barriers to delivery of IPTp have been shown to be relatively consistent across countries [12]. Further, preliminary analyses of separate regression models for each country, where confidence intervals of the estimates were overlapping, suggest that there was no significant effect modification by country (Additional file 2). All analyses were performed using the $\mathrm{R}$ statistical software [36], and the 'geepack' package [37] was used to fit the modified Poisson regression models with clustered standard errors.

In order to quantify the contribution of selected potentially modifiable determinants of IPTp delivery (defined as the provision of any dose of IPTp-DOT), the population attributable fraction (PAF) was estimated. The PAF quantifies the proportional increase in IPTp delivery during ANC consultations that would have occurred if a barrier to delivery had been completely removed or a driver fully scaled-up. The following equation was used to compute this metric:

$$
\mathrm{PAF}=\frac{R R-\left[P_{0}+P_{1}(R R)\right]}{P_{0}+P_{1}(R R)}
$$

where $R R$ is the relative risk for the selected determinant; $P_{O}$ is the proportion of the currently non-exposed population; and $P_{1}$ is the proportion of the currently exposed population. The exposed and unexposed proportions of the population were calculated using the appropriate sampling weights. These weights were multiplied by the number of reported ANC visits per month for each facility to obtain estimates representative of all women seeking ANC services. The five surveys were combined and each survey was re-weighted proportionally to the size of its country's female population. For cases where the determinant was a barrier (i.e., $R R<1$ ), the coding of the exposure was reversed so that the reference exposure (non-exposed) is changed to the exposed and the inverse of the RR was inputted in the modified PAF formula. Uncertainty intervals (UI) for this modified version of the PAF were obtained using 10,000 Monte Carlo simulations.

Finally, the joint contribution of multiple modifiable determinants of IPTp delivery, taking into account their potential correlation, was assessed. Because preliminary analyses have shown that the selected determinants do not interact on the multiplicative scale, their joint contribution was estimated using a multiplicative excess risk scale. The joint PAF for multiple determinants was computed by summing the combined $R R$ of individual records using the appropriate survey weights [38]. This joint PAF was calculated using the following formula:

$$
\begin{gathered}
\text { Joint PAF }=\frac{\sum R R_{i}{ }^{\prime}-\sum R R_{i}}{\sum R R_{i}} \\
\text { where } R R_{\mathrm{i}}{ }^{\prime}=\exp \left[\sum_{j=1}^{n} \beta_{j}\left(X_{i j}{ }^{\prime}\right)\right] \text { and } R R_{\mathrm{i}}=\exp \left[\sum_{j=1}^{n} \beta_{j}\left(X_{i j}\right)\right]
\end{gathered}
$$

where $\beta_{j}$ corresponds to the log RR per unit of exposure of determinants $j ; X_{i j}$ ' is a vector that contains the alternative distribution of determinants $j$ for each record $i$ (for this study, binary determinants were set to 1 , corresponding to the situation were all providers would be exposed); and $X_{i j}$ is the current distribution of determinant $j$ for each record $i$. As for the individual PAF, the coding of barriers (i.e., $\mathrm{RR}<1$ ) was reversed and the inverse of the RR was used. To calculate uncertainty intervals for the joint PAF, 10,000 log-RR from a multivariate normal distribution were first simulated. These Monte Carlo simulations were then combined with 10,000 bootstrap replicates of the current exposure distribution, implicitly modelling the correlation structure of the different exposures and defining the sampling unit at the 
facility level to take into account clustering of observations. These analyses were also performed using the $\mathrm{R}$ statistical software [36].

\section{Results}

A total of 2,746 health facilities were surveyed in the five combined SPA surveys, of which 2,200 provided ANC services (Figure 1). Among these, 1,577 were offering this type of services on the day of the survey and 1,310 health facilities were further selected for the maternal health subsurvey with observations of client-provider's ANC consultation and client exit interviews. After excluding observations of consultations performed in nonmalarious areas of Namibia $(n=190)$, those for women less than 16 weeks into their pregnancy $(n=251)$, and those for which the main IPTp outcome was missing $(n=55)$, a total of 4,976 observations of client-provider consultations contributed information to the analyses. These consultations were performed in 1,285 different facilities by 1,438 unique providers. The great majority of facilities (89.9\%) had only one provider contributing information and each provider had an average of 3.5 observed ANC consultations.

The characteristics of facilities, providers and clients are described in Table 1. Anti-malarial prophylaxis was administered as DOT in only $35 \%$ of all consultations. There were important variations in the proportion of different types of facilities surveyed between countries, reflecting national differences in health system delivery of ANC services. For example, $94.6 \%$ of facilities providing ANC services in Rwanda were health centres, but this proportion dropped to $8.3 \%$ in Tanzania. Namibia's health system is almost entirely public and less than $1 \%$ of facilities in malarious areas of this country charged user-fees for ANC medicines. In contrast, private practices and user fees were more common in Kenya. Overall, 93\% of the surveyed facilities claimed to routinely provide IPTp as part of their ANC services but this proportion varied from a low of $73 \%$ in Namibia to a high of $99 \%$ in Uganda. SP was not available in $11 \%$ of the facilities on the day of the survey. The great majority of providers of ANC services were either enrolled nurse/ midwife $(36 \%)$ or registered nurse/midwife $(42 \%)$, and only $26 \%$ of all providers had received IPTp training in the year preceding the survey.

Results from univariate and multivariable regressions (Figure 2) show that hospitals and health centres did not differ much in their propensity to deliver IPTp but health posts and dispensary were $20 \%$ less likely to deliver IPTp as compared to health centres. Providers

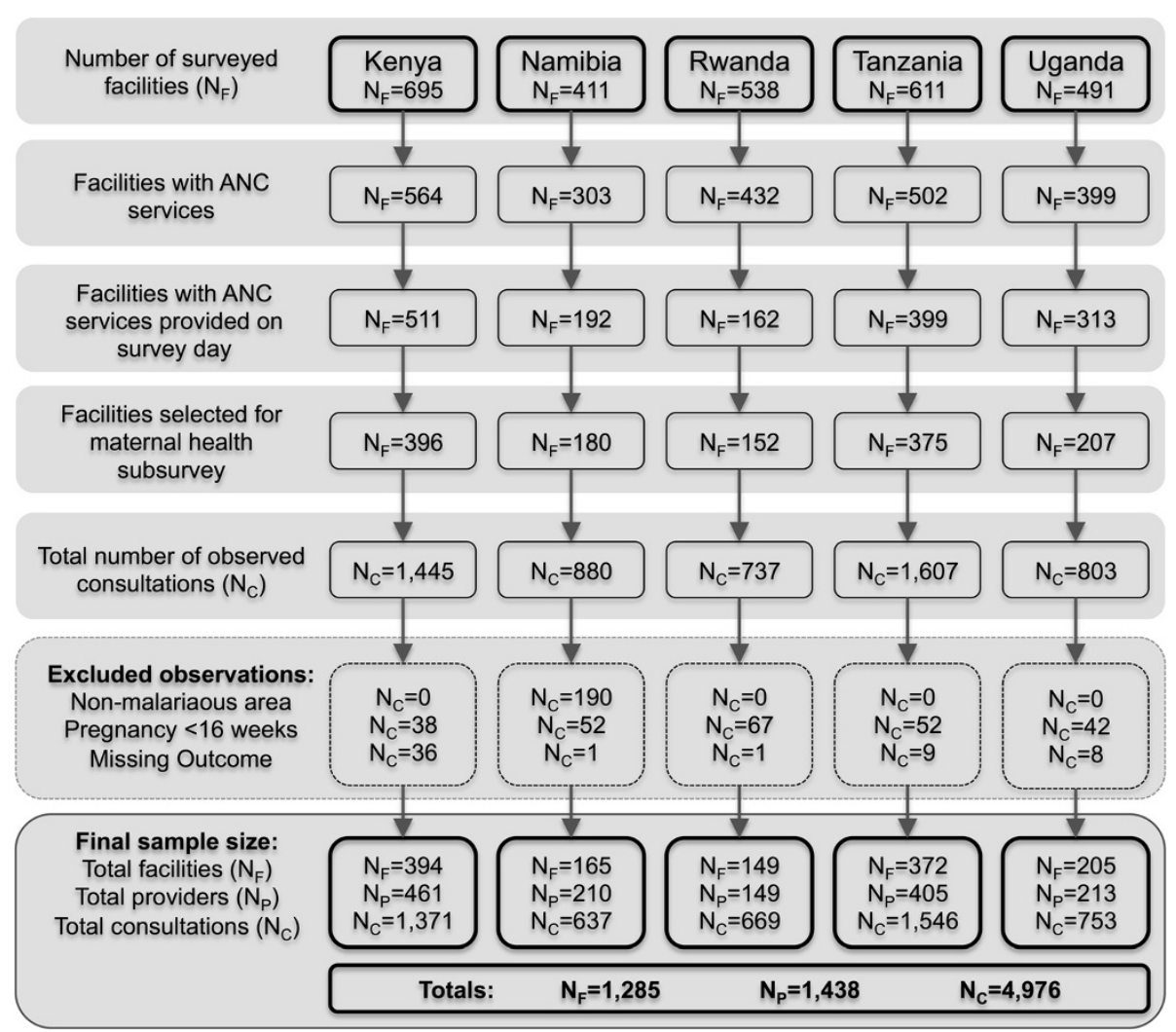

Figure 1 Flowchart of the inclusion of the health facilities, providers, and clients of ANC services in the service provision assessments survey of Kenya, Namibia, Rwanda, Tanzania, and Uganda (2006-2010). 
Table 1 Characteristics of facilities, providers and of the observed women's antenatal care consultations (if $\geq 16$ weeks pregnant) for five service provision assessment surveys conducted in sub-Saharan Africa

\begin{tabular}{|c|c|c|c|c|c|c|}
\hline Variables & Kenya 2010 & Namibia 2009 & Rwanda 2007 & Tanzania 2006 & Uganda 2007 & All combined \\
\hline Facility & $N=394$ & $N=165$ & $N=149$ & $N=372$ & $N=205$ & $N=1,285$ \\
\hline \multicolumn{7}{|l|}{ Facility type } \\
\hline Health centre & $25.6 \%$ & $19.4 \%$ & $94.6 \%$ & $8.3 \%$ & $27.3 \%$ & $28.1 \%$ \\
\hline Hospital & $52.0 \%$ & $2.4 \%$ & $2.7 \%$ & $30.1 \%$ & $41.0 \%$ & $31.8 \%$ \\
\hline Health post/dispensary & $22.3 \%$ & $78.2 \%$ & $2.7 \%$ & $61.6 \%$ & $31.7 \%$ & $40.1 \%$ \\
\hline Public facility & $67.3 \%$ & $99.4 \%$ & $70.5 \%$ & $80.4 \%$ & $77.1 \%$ & $77.1 \%$ \\
\hline Facility has fee for medicines & $31.7 \%$ & $0.6 \%$ & $4.7 \%$ & $4.3 \%$ & $3.9 \%$ & $12.2 \%$ \\
\hline Missing & $0.5 \%$ & $0.0 \%$ & $5.4 \%$ & $0.0 \%$ & $0.5 \%$ & $0.9 \%$ \\
\hline Facility has IPTp guidelines & $53.0 \%$ & $3.0 \%$ & $50.3 \%$ & $51.3 \%$ & $47.8 \%$ & $45.0 \%$ \\
\hline Facility claims routine IPTp & $95.2 \%$ & $72.7 \%$ & $96.0 \%$ & $94.6 \%$ & $99.5 \%$ & $92.9 \%$ \\
\hline Missing & $0.0 \%$ & $0.0 \%$ & $0.0 \%$ & $0.5 \%$ & $0.0 \%$ & $0.2 \%$ \\
\hline SP stocked-out on visit day & $8.6 \%$ & $22.4 \%$ & $10.7 \%$ & $12.1 \%$ & $6.8 \%$ & $11.4 \%$ \\
\hline Missing & $0.8 \%$ & $0.6 \%$ & $0.0 \%$ & $0.8 \%$ & $0.0 \%$ & $0.5 \%$ \\
\hline Provider & $\mathrm{N}=461$ & $N=210$ & $N=149$ & $N=405$ & $N=213$ & $N=1,438$ \\
\hline \multicolumn{7}{|l|}{ Type of provider } \\
\hline Physician & $5.9 \%$ & $0.0 \%$ & $2.0 \%$ & $5.7 \%$ & $3.3 \%$ & $4.2 \%$ \\
\hline Enrolled nurse/midwife & $47.5 \%$ & $59.5 \%$ & $2.7 \%$ & $14.1 \%$ & $50.2 \%$ & $35.6 \%$ \\
\hline Registered nurse/midwife & $42.7 \%$ & $38.6 \%$ & $79.2 \%$ & $34.6 \%$ & $33.8 \%$ & $42.3 \%$ \\
\hline Other & $2.4 \%$ & $1.0 \%$ & $15.4 \%$ & $44.4 \%$ & $7.0 \%$ & $16.1 \%$ \\
\hline Missing & $1.5 \%$ & $1.0 \%$ & $0.7 \%$ & $1.2 \%$ & $5.6 \%$ & $1.9 \%$ \\
\hline Supervised in last 6 months & $78.3 \%$ & $70.5 \%$ & $86.6 \%$ & $78.8 \%$ & $79.3 \%$ & $78.3 \%$ \\
\hline Missing & $1.7 \%$ & $1.0 \%$ & $1.3 \%$ & $1.2 \%$ & $6.1 \%$ & $2.1 \%$ \\
\hline Trained for IPTp in last year & $31.2 \%$ & $25.7 \%$ & $30.2 \%$ & $16.5 \%$ & $32.4 \%$ & $26.3 \%$ \\
\hline Missing & $2.2 \%$ & $1.0 \%$ & $0.7 \%$ & $1.2 \%$ & $6.1 \%$ & $2.2 \%$ \\
\hline Consultations/clients & $N=1,371$ & $N=637$ & $N=669$ & $N=1,546$ & $N=753$ & $N=4,976$ \\
\hline IPTp administered as DOT & $41.8 \%$ & $8.0 \%$ & $59.0 \%$ & $25.4 \%$ & $42.0 \%$ & $34.7 \%$ \\
\hline Prescribed/Given IPTp & $67.5 \%$ & $19.1 \%$ & $69.3 \%$ & $49.1 \%$ & $65.2 \%$ & $55.4 \%$ \\
\hline Missing & $0.2 \%$ & $0.3 \%$ & $1.0 \%$ & $0.1 \%$ & $0.5 \%$ & $0.3 \%$ \\
\hline Primigravidae & $31.1 \%$ & $35.2 \%$ & $28.7 \%$ & $24.3 \%$ & $30.5 \%$ & $29.1 \%$ \\
\hline Missing & $1.0 \%$ & $0.2 \%$ & $0.6 \%$ & $0.1 \%$ & $0.3 \%$ & $0.4 \%$ \\
\hline First visit at facility & $38.9 \%$ & $50.7 \%$ & $50.2 \%$ & $39.4 \%$ & $46.6 \%$ & $43.2 \%$ \\
\hline Missing & $0.1 \%$ & $0.0 \%$ & $0.0 \%$ & $0.0 \%$ & $0.0 \%$ & $0.0 \%$ \\
\hline \multicolumn{7}{|l|}{ Education } \\
\hline None & $8.2 \%$ & $11.9 \%$ & $35.1 \%$ & $21.9 \%$ & $19.8 \%$ & $18.3 \%$ \\
\hline Primary & $49.9 \%$ & $25.0 \%$ & $54.9 \%$ & $66.7 \%$ & $48.5 \%$ & $52.4 \%$ \\
\hline Secondary/Higher & $39.2 \%$ & $63.1 \%$ & $7.8 \%$ & $11.3 \%$ & $29.0 \%$ & $27.8 \%$ \\
\hline Missing & $2.7 \%$ & $0.0 \%$ & $2.2 \%$ & $0.1 \%$ & $2.8 \%$ & $1.5 \%$ \\
\hline \multicolumn{7}{|l|}{ Age } \\
\hline$<20$ years & $13.7 \%$ & $21.5 \%$ & $4.0 \%$ & $15.5 \%$ & $21.1 \%$ & $15.1 \%$ \\
\hline 20-29 years & $63.4 \%$ & $49.5 \%$ & $59.2 \%$ & $57.8 \%$ & $56.8 \%$ & $58.3 \%$ \\
\hline$\geq 30$ years & $19.9 \%$ & $25.7 \%$ & $34.4 \%$ & $26.6 \%$ & $19.1 \%$ & $24.6 \%$ \\
\hline Missing & $3.0 \%$ & $3.3 \%$ & $2.4 \%$ & $0.2 \%$ & $2.9 \%$ & $2.1 \%$ \\
\hline Weeks of pregnancy (mean) & 29.9 & 27.9 & 26.5 & 27.9 & 28.8 & 28.4 \\
\hline
\end{tabular}

Descriptive statistics do not take into account survey weights.

$\mathrm{ANC}=$ antenatal care; IPTp = intermittent preventive treatment for malaria in pregnancy; DOT $=$ directly observed therapy. 


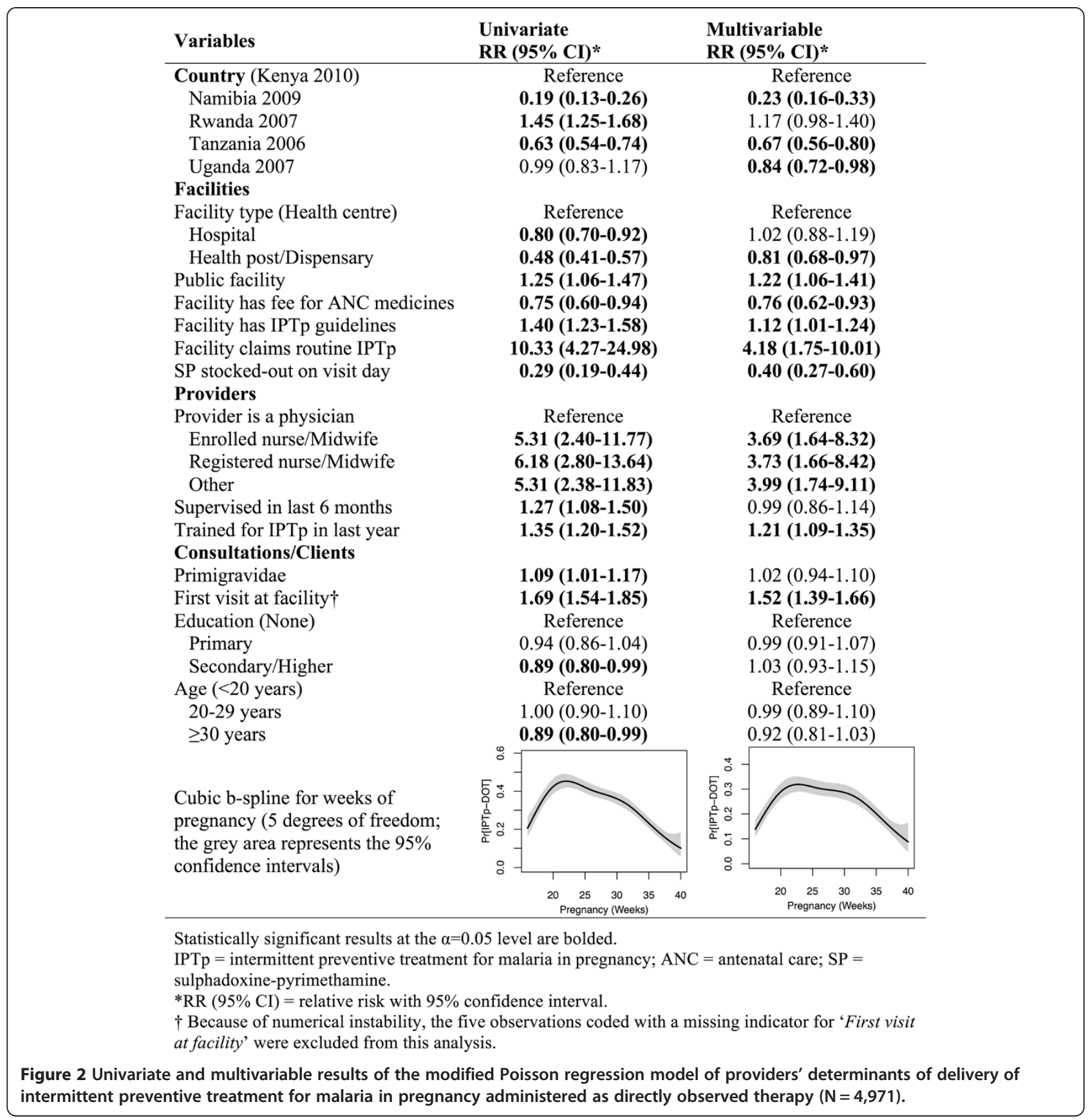

working in public facilities are, however, $22 \%$ more likely to provide anti-malarial prophylaxis to their ANC clients. Importantly, user-fees for ANC medicines seem to deter client from receiving IPTp in both univariate and multivariable analyses with an adjusted RR of 0.76 (95\% confidence interval (95\% CI): 0.62-0.93). Having IPTp guidelines or protocols in the facilities was also associated with increased delivery of this intervention $(\mathrm{RR}=1.12 ; 95 \% \mathrm{CI}: 1.01-1.24)$. Unsurprisingly, the factor whose effect was greatest was whether the facility claimed that IPTp was routinely offered as part of their
ANC services. Such facilities were close to four times more likely to deliver this intervention. An important barrier to IPTp was stock-outs of SP on the day of the survey. Consultations occurring in stocked-out facilities were $60 \%$ less likely $(\mathrm{RR}=0.40 ; 95 \% \mathrm{CI}: 0.27-0.60)$ to deliver IPTp. Even if SP was stocked-out in the facility's inventory on the day of the survey, providers could have administered IPTp if a separate supply was kept in the office were ANC consultations occurred, explaining why some women still received IPTp in stocked-out facilities. 
At the provider level, clinicians were the least likely to deliver the IPTp intervention. Providers with other qualifications did not exhibit much difference in their estimated effect size measures. Providers who had been supervised in the previous six months were not more likely to deliver IPTp during a consultation. In contrast, providers who have received IPTp training during the previous year were $21 \%$ more likely to give anti-malarial prophylaxis during ANC visits (RR: 1.21; 95\% CI: 1.09-1.35). Providers whose clients were seeking ANC services for the first time in their current pregnancy were $52 \%(\mathrm{RR}=1.52 ; 95 \% \mathrm{CI}$ : 1.39-1.66) more likely to give them IPTp while age of the client was not statistically significant in the multivariable analysis. Finally, clients in their $20^{\text {th }}$ to $32^{\text {nd }}$ week of pregnancy were the most likely to receive IPTp.

All these results were robust to different model specifications. Specifically, the different effect size measures were robust to the inclusion of 60 dummy variables representing the different subnational regions (Additional file 3). Including fixed effects at the regional level could effectively account for unmeasured differences in malaria endemicity levels or regional targeting by national malaria control programmes. Further, restricting the analyses to consultations from providers working in facilities where IPTp was claimed to be routinely offered as part of their ANC services had no impact on the other determinants' point estimates (Additional file 4). Finally, when the outcome was defined as providers prescribing or giving antimalarial prophylaxis - without considering if it was administered as DOT - all determinants remained statistically significant (except for the availability of IPTp guidelines/protocols), although the point estimates were generally closer to the null (data not shown).

To get a measure of potential impact, the proportional increase in IPTp delivery that would occur if selected barriers would have been removed or if drivers would have been fully scaled was estimated. Among the covariates that were found to be significant in the multivariable regression model, the following determinants were considered potentially modifiable: 1 ) removing user-fees for ANC medicines; 2) providing all facilities with IPTp guidelines/protocols; 3) implementing IPTp as part of the routine ANC services in all facilities; 4) preventing stock-outs of SP; and, 5) providing annual IPTp-specific training to ANC providers. As such, providers' qualifications and client-level determinants such as education, age, weeks of pregnancy, or primigravidae status are not considered modifiable.

The modified PAF showed that, if the estimated effect size measures have a causal interpretation, removing userfees for ANC medicines would have a negligible impact on delivery of IPTp (Table 2). This is not entirely surprising since the great majority of facilities in the five surveyed countries were not applying any charges. Similarly,

\section{Table 2 Proportional increase attributable to selected modifiable determinants of intermittent preventive treatment for malaria in pregnancy delivery}

\begin{tabular}{lll}
\hline Barriers/Drivers & $\begin{array}{l}\text { Current } \\
\text { prevalence* }\end{array}$ & $\begin{array}{l}\text { Proportional increase } \\
\text { in IPTp (95\% UI) }\end{array}$ \\
\hline $\begin{array}{l}\text { Removing user-fees for ANC } \\
\text { medicines }\end{array}$ & $12.2 \%$ & $3.1 \%(1.4-6.5 \%)$ \\
$\begin{array}{l}\text { Providing IPTp guidelines to } \\
\text { all facilities }\end{array}$ & $50.1 \%$ & $5.5 \%(1.9-15.1 \%)$ \\
$\begin{array}{l}\text { Integrating IPTp in routine } \\
\text { ANC services }\end{array}$ & $96.0 \%$ & $3.2 \%(1.8-5.2 \%)$ \\
$\begin{array}{l}\text { Preventing stock-outs of SP } \\
\text { Annual IPTp-training for } \\
\text { providers }\end{array}$ & $10.7 \%$ & $6.8 \%(4.1-10.4 \%)$ \\
Joint effect & $30.2 \%$ & $13.9 \%(7.5-24.8 \%)$
\end{tabular}

$\mathrm{UI}=$ uncertainty intervals; SP = sulphadoxine-pyrimethamine; IPTp = intermittent preventive treatment for malaria in pregnancy; $\mathrm{ANC}=$ antenatal care. *Prevalence estimates take into account the appropriate survey weights that were multiplied by the facility's number of ANC visits per month to represent the distribution of consultations for which the barrier/driver was present.

providing IPTp guidelines/protocols to facilities would result in a $5.5 \%$ increase in IPTp delivery. Implementing routine IPTp in all facilities would increase by only $3.2 \%$ the delivery of IPTp during consultations, as $96 \%$ of facilities already offered IPTp as part of their ANC services. Preventing SP stock-outs would have a more important impact, increasing delivery of this intervention by $6.8 \%$. These analyses suggest, however, that annual training of ANC providers for IPTp would have the biggest impact on IPTp delivery with an estimated $13.9 \%$ increase. Overall, addressing these five barriers/drivers jointly would result in a $30.6 \%$ (95\% UI: $20.7-44.2 \%$ ) increase in the proportion of client consultations where anti-malarial prophylaxis would have been administered as DOT.

\section{Discussion}

ANC attendance in sub-Saharan Africa has been referred to as a success story [39]. Among the five countries analysed in this paper, coverage for at least two ANC visits approximated 90\% (Table 3). Coverage of IPTp, however, was much lower, varying between 11 and 27\% according to demographic and health survey data. As current guidelines recommend a minimum of four antenatal visits, this IPTp gap represents considerable missed opportunities to deliver a cost-effective intervention. This paper has shown that the main potentially modifiable determinants of providers' delivery of IPTp were the absence of user-fees for ANC medicines, availability of IPTp-specific guidelines/ protocols in the facility, whether a facility claimed to routinely offer IPTp as part of their ANC services, the absence of SP stock-outs, and providers being recently trained for IPTp. The respective impact of these determinants on the expected proportional increase in IPTp delivery varied importantly, however. Training providers 
Table 3 Mothers' attendance of antenatal care and uptake of IPTp in the five selected countries

\begin{tabular}{|c|c|c|c|c|c|c|}
\hline Country & Sample size & $\geq 1$ ANC visit & $\geq 2$ ANC visits & $\geq 1$ dose of SP & $\geq 2$ doses of SP & Reference \\
\hline Kenya & 3,973 and 2,264 & $92.7 \%$ & $88.4 \%$ & $35.5 \%$ & $15.1 \%$ & DHS 2008-09 [40] \\
\hline Namibia & 3,898 and 2,054 & $96.2 \%$ & $94.5 \%$ & $27.8 \%$ & $10.6 \%$ & DHS 2006-07 [41] \\
\hline Rwanda & 3,658 and 2,267 & $97.3 \%$ & $91.4 \%$ & $53.0 \%$ & $17.7 \%$ & DHS 2007-08 [42] \\
\hline Tanzania & 5,519 and 3,266 & $98.0 \%$ & $94.4 \%$ & $63.3 \%$ & $27.2 \%$ & DHS 2010 [43] \\
\hline Uganda & 4,958 and 3,092 & $95.7 \%$ & $91.7 \%$ & $48.4 \%$ & $26.7 \%$ & DHS 2011 [44] \\
\hline
\end{tabular}

ANC = antenatal care; SP = sulphadoxine-pyrimethamine; DHS = demographic and health survey.

*The first listed sample size corresponds to the denominator used to calculate ANC attendance and the second to the denominator use to estimate coverage of IPTp with SP. The sample sizes differ because the recall period for ANC attendance and IPTp coverage is for the most recent live birth over the last five years versus the last two years, respectively.

for IPTp would have the most impact with an expected $14 \%$ increase in delivery during ANC consultations. Addressing these five barriers/drivers jointly would increase the proportion of consultations where IPTp is delivered by $31 \%$. These estimates depend strongly on the current distribution of these determinants, however, and countries where barriers are more common should expect higher impact (country-specific estimates can be found in Additional file 5).

The fact that addressing these five determinants jointly would only lead to a $31 \%$ increase in IPTp delivery warrants further discussion as it emphasizes that no single determinant is responsible for the IPTp gap. Other factors, not measured in SPA surveys, could be important barriers to IPTp delivery. These include the unavailability of potable drinking water to swallow the SP tablets and/or sharing of drinking cups among clients [45,46]; providers having high workload, low motivation, and/or being poorly organized [46-48]; providers' confusion about timing and number of IPTp doses [11,23]; women's previous experiences of SP-related side effects and/or fear of side effects $[49,50]$; women refusing to take the tablets on an empty stomach $[12,45]$; and, women's perception of malaria risk and their lack of knowledge about the benefits of IPTp [12,51,52]. Although the last three barriers do not correspond to supply-side determinants, providers could help mitigate/overcome them by providing women with appropriate information during their ANC consultation on the role, innocuity, and importance of IPTp.

Among the determinants that were not deemed to be modifiable, it was found that publicly managed facilities had higher rates of IPTp delivery than private ones. This could result from IPTp guidelines being easier to implement in public facilities as they are under the direct control of national authorities. The consequence being, as was observed in Nigeria, that providers from private facilities have incorrect knowledge of IPTp recommendations [53]. Further, local health authorities in Tanzania acknowledged that preferential treatment was provided to public facilities when it came to budget allocation for IPTp as they feared commercialization of this health service in private facilities [54].
The data showed that providers were $52 \%$ more likely to deliver IPTp if this was the client's first ANC visit at the facility for their current pregnancy (conditional on being 16 weeks pregnant or more). Unclear policy and guidance for IPTp has been identified as a key barrier to effective delivery of this intervention and providers' confusion about timing of the second SP dose could explain this result $[12,55]$. In addition, providers were less likely to provide IPTp to women outside the $20^{\text {th }}$ to $32^{\text {nd }}$ weeks of pregnancy range. Tanzania and Namibia used an IPTp schedule that differed from WHO recommendations and this could have contributed to the observed pattern [56,57] (i.e., administering IPTp between 20-24 and 28-32 weeks of gestation for Tanzania and between 26-28 and 34-36 weeks of gestation for Namibia).

Clinicians were the least likely to deliver IPTp to their clients. In ANC settings, these providers generally dispense palliative care for symptomatic pregnant women where IPTp might be contraindicated. Alternatively, it is also possible that nurses and midwives are more cognizant of the recommended standards of care and correctly apply the IPTp protocols. The fact that supervision of providers, a proxy for improved management practices, was not found to impact delivery of the intervention should not be taken at face value as the estimates could be affected by reverse causality. That is, underperforming providers that do not follow guidelines might attract supervisory attention.

This study has a number of limitations. First, several demand-side determinants of IPTp uptake were not collected by the SPA surveys. These include, for example, individual malaria risk perceptions and knowledge of benefits of anti-malarial prophylaxis during pregnancy. Although the omission of such important determinants contributes to increasing the standard errors of the estimates, they are likely not correlated to provider's characteristics (conditional on the covariates included in the model) and should not bias the results. Second, information on the number and timing of the preceding dose(s) of SP, if any, was not available. The guidelines in effect at the time of the surveys recommended two doses of SP during the second and third trimester of pregnancy - 
with the exception of Kenya, which in 2006 recommended administration of IPTp at each scheduled ANC visits after quickening [58]. Hence, some of the clients could have been ineligible for IPTp if they had already received their two doses or if they had received their first one within the preceding four weeks. Coverage of two or more doses of IPTp is rather low in these countries (Table 3), however, and the likelihood that women were eligible for a third dose is small. Further, the four recommended ANC visits are usually scheduled at least one month apart, which would have also minimized the number of women ineligible for IPTp because their preceding dose was administered within the proscribed period. Another scenario for which women might have been ineligible for IPTp consists of SP being contra-indicated in HIV-infected women receiving co-trimoxazole (CTX) prophylaxis (but not those taking antiretroviral therapy). The proportion of women ineligible for IPTp based on CTX prophylaxis should be small, however, as implementation of this intervention has been slow in resource-limited settings [59]. Third, providers knew that they were being observed and the Hawthorn effect [60] could have affected the outcomes of ANC consultations. The most likely direction of this effect is that providers would have been more prone to administer anti-malarial prophylaxis, in which case the proportion of ANC consultations for which IPTp is given is probably lower than that reported in the surveys. However, if this effect is not correlated with the characteristics of the facility, the provider, or the client, then the effect size estimates should not be biased. Lastly, the proportional increase estimates derived from the PAF calculations assumes that the effect size measures have a causal interpretation. The lack of exogenous variation for the selected modifiable determinants prohibits such a strong causal claim. Yet, the estimates were robust to different model specifications and these determinants have been identified previously in both qualitative and quantitative studies [12,15,21,23,48,53]. By combining information on exposure and effect size, it is believed that the PAF calculations shed an important light on the types of interventions most likely to increase coverage of IPTp.

This study is believed to be the first to use nationally representative survey data to assess determinants of provider's delivery of IPTp during ANC consultations and to quantify the potential impact of these determinants. The use of standardized survey instruments enabled the pooling of data from surveys conducted in 1,285 facilities located in five countries. In addition, in comparison to other studies, an objectively measured outcome was used in lieu of women's selfreport of IPTp uptake (which is often affected by recall bias) [61].

\section{Conclusion}

This study highlights a number of potential interventions to increase coverage of IPTp. User-fees for ANC medicines and facilities not having implemented routine IPTp are two important barriers to IPTp delivery, but these were relatively uncommon in the five surveyed countries. Close to half of facilities lack IPTp-specific guidelines/protocols but providing such items to all facilities would not increase delivery of this intervention substantially. In contrast, interventions aiming at improving the supply-chain management for SP to prevent stock-outs would have some impact, although modest, on delivery of IPTp. A quasi-experimental study conducted in the Zambia has shown that simple structural and information flow changes can lead to important improvements in the availability of drugs [62]. It is doubtful, however, that SP stock-outs could be entirely eliminated, especially in countries where parts of health budgets are contingent upon external funding [63]. As such, the proportional increase in IPTp delivery that was estimated should be interpreted as a theoretical upper bound. One of the most interesting findings of this study was that training of providers could lead to important increases in IPTp delivery. Only 30\% of ANC consultations were offered by providers who had received IPTp training during the year before the survey, leaving room for improvements.

Interventions aimed at increasing knowledge and competency of health workers are available and have shown positive impacts on delivery of IPTp in Uganda [21] and Kenya [23]. Future studies should evaluate the potential of targeting interventions, either spatially in areas of low IPTp coverage or to specific facilities. Private facilities, health post and dispensary were shown to be less likely to administer IPTp and should be prioritized to increase coverage of this intervention. Multi-pronged approaches are most likely to yield substantial increases in IPTp coverage, and addressing providers' barriers to effective IPTp delivery is urgently required if the Roll Back Malaria Partnership goal of $100 \%$ coverage by 2015 [13] and the President's Malaria Initiative target of $85 \%$ coverage [14] are to be achieved.

\section{Additional files}

\footnotetext{
Additional file 1: Harmonization of the classification schemes for type of facility and the provider's qualification. This table provides information on the different classification schemes for facility types and provider's qualifications were harmonized to be used in the pooled analysis.

Additional file 2: Country-specific multivariable results of the modified Poisson regression models of providers' determinants of delivery of intermittent preventive treatment for malaria in pregnancy administered as directly observed therapy. Presented in this table are the multivariable results when the regression models were run separately for Kenya, Namibia, Rwanda, Tanzania, and Uganda.
} 
Additional file 3: Multivariable results of the modified Poisson regression models, with fixed effect at the regional level, of providers' determinants of delivery of intermittent preventive treatment for malaria in pregnancy (IPTp) administered as directly observed therapy. This regression model controls for 60 dummy variables that correspond to the different regions of the five surveyed countries. Controlling for these regional variables had no effect on the main determinants of IPTp delivery.

Additional file 4: Multivariable results of the modified Poisson regression models of providers' determinants of delivery of intermittent preventive treatment for malaria in pregnancy administered as directly observed therapy (IPTp) in facilities that claim that this intervention is routinely offered as part of their antenatal care services (ANC). Facilities that do not claim that IPTP is part of their routine ANC services were excluded. Results show that this has no impact on the main determinants of IPTp delivery.

Additional file 5: Proportional increase attributable to selected modifiable determinants of intermittent preventive treatment for malaria in pregnancy (IPTp, administered as directly observed therapy) for Kenya, Namibia, Rwanda, Tanzania, and Uganda. The proportional increase in IPTp delivery was calculated using the population attributable fraction for each country separately.

\section{Competing interests}

The authors declare that they have no competing interests.

\section{Authors' contributions}

MCC and MMG developed the original research idea for the paper and formulated the research question. MMG managed the SPA databases, performed the statistical analyses and interpreted the results under the supervision of MCC. MMG drafted the manuscript that MCC subsequently edited. Both authors reviewed the paper for intellectual content and approved the final manuscript.

\section{Acknowledgements}

MMG's research is supported by a Doctoral Foreign Study Award from the Canadian Institutes of Health Research and an International Fulbright Science \& Technology Award, sponsored by the Bureau of Educational and Cultural Affairs of the US Department of State. The funders had no role in study design, data collection and analysis, decision to publish, or preparation of the manuscript.

Received: 29 June 2014 Accepted: 4 November 2014

Published: 20 November 2014

\section{References}

1. Desai M, ter Kuile FO, Nosten F, McGready R, Asamoa K, Brabin B, Newman $\mathrm{RD}$ : Epidemiology and burden of malaria in pregnancy. Lancet Infect Dis 2007, 7:93-104.

2. Dellicour S, Tatem AJ, Guerra CA, Snow RW, ter Kuile FO: Quantifying the number of pregnancies at risk of malaria in 2007: a demographic study. PLoS Med 2010, 7:e1000221.

3. WHO: WHO Policy Brief for the Implementation of Intermittent Preventive Treatment of Malaria in Pregnancy using Sulfadoxine-Pyrimethamine (IPTp-SP). Geneva, Switzerland: World Health Organization (WHO) Global Malaria Programme, WHO Department of Reproductive Health and Research, and WHO Department of Maternal, Newborn, Child and Adolescent Health; 2013:12.

4. Garner P, Gülmezoglu AM: Drugs for preventing malaria in pregnant women. Cochrane Database Syst Rev 2006, 18:CD000169.

5. Eisele TP, Larsen DA, Anglewicz PA, Keating J, Yukich J, Bennett A, Hutchinson P, Steketee RW: Malaria prevention in pregnancy, birthweight, and neonatal mortality: a meta-analysis of 32 national cross-sectional datasets in Africa. Lancet Infect Dis 2012, 12:942-949.

6. Menéndez C, Bardají A, Sigauque B, Sanz S, Aponte JJ, Mabunda S, Alonso PL: Malaria prevention with IPTp during pregnancy reduces neonatal mortality. PLoS One 2010, 5:e9438.

7. Eisele TP, Larsen D, Steketee RW: Protective efficacy of interventions for preventing malaria mortality in children in Plasmodium falciparum endemic areas. Int J Epidemiol 2010, 39(Suppl 1):i88-i101.
8. ter Kuile FO, van Eijk AM, Filler SJ: Effect of sulfadoxine-pyrimethamine resistance on the efficacy of intermittent preventive therapy for malaria control during pregnancy: a systematic review. JAMA 2007, 297:2603-2616.

9. Sicuri E, Bardají A, Nhampossa T, Maixenchs M, Nhacolo A, Nhalungo D, Alonso PL, Menéndez C: Cost-effectiveness of intermittent preventive treatment of malaria in pregnancy in southern Mozambique. PLoS One 2010, 5:e13407.

10. van Eijk AM, Hill J, Alegana VA, Kirui V, Gething PW, ter Kuile FO, Snow RW: Coverage of malaria protection in pregnant women in sub-Saharan Africa: a synthesis and analysis of national survey data. Lancet Infect Dis 2011, 11:190-207.

11. van Eijk AM, Hill J, Larsen DA, Webster J, Steketee RW, Eisele TP, Ter Kuile FO: Coverage of intermittent preventive treatment and insecticidetreated nets for the control of malaria during pregnancy in sub-Saharan Africa: a synthesis and meta-analysis of national survey data, 2009-11. Lancet Infect Dis 2013, 13:1029-1042.

12. Hill J, Hoyt J, van Eijk AM, D'Mello-Guyett L, Ter Kuile FO, Steketee R, Smith H, Webster J: Factors affecting the delivery, access, and use of interventions to prevent malaria in pregnancy in sub-Saharan Africa: a systematic review and meta-analysis. PLoS Med 2013, 10:e1001488,

13. RBM: Refined/Updated Global Malaria Action Plan Objectives, Targets, Milestones and Priorities Beyond 2011. Geneva, Switzerland: Roll Back Malaria Partnership; 2011:6

14. PMI: The President's Malaria Initiative - Seventh Annual Report to Congress. Washington, DC: United States Agency for International Development, Centers for Disease Control and Prevention, Department of Health \& Human Services; 2013:53.

15. Crawley J, Hill J, Yartey J, Robalo M, Serufilira A, Ba-Nguz A, Roman E, Palmer A, Asamoa K, Steketee R: From evidence to action? Challenges to policy change and programme delivery for malaria in pregnancy. Lancet Infect Dis 2007, 7:145-155.

16. WHO: Updated WHO Policy Recommendation - Intermittent Preventive Treatment of Malaria in Pregnancy using Sulfadoxine-Pyrimethaine (IPTp-SP). Geneva, Switzerland: World Health Organization, Global Malaria Programme; 2012:2.

17. Okeibunor JC, Orji BC, Brieger W, Ishola G, Otolorin E, Rawlins B, Ndekhedehe EU, Onyeneho N, Fink G: Preventing malaria in pregnancy through community-directed interventions: evidence from Akwa lbom State. Nigeria Malar J 2011, 10:227.

18. Ndyomugyenyi R, Tukesiga E, Katamanywa J: Intermittent preventive treatment of malaria in pregnancy (IPTp): participation of communitydirected distributors of ivermectin for onchocerciasis improves IPTp access in Ugandan rural communities. Trans R Soc Trop Med Hyg 2009, 103:1221-1228.

19. Msyamboza KP, Savage EJ, Kazembe PN, Gies S, Kalanda G, D'Alessandro U, Brabin BJ: Community-based distribution of sulfadoxine-pyrimethamine for intermittent preventive treatment of malaria during pregnancy improved coverage but reduced antenatal attendance in southern Malawi. Trop Med Int Health 2009, 14:183-189.

20. Mbonye AK, Bygbjerg IC, Magnussen P: A community-based delivery system of intermittent preventive treatment of malaria in pregnancy and its effect on use of essential maternity care at health units in Uganda. Trans R Soc Trop Med Hyg 2007, 101:1088-1095.

21. Mbonye AK, Yanow S, Birungi J, Magnussen P: A new strategy and its effect on adherence to intermittent preventive treatment of malaria in pregnancy in Uganda. BMC Pregnancy Childbirth 2013, 13:178.

22. Gies S, Coulibaly SO, Ky C, Ouattara FT, Brabin BJ, D'Alessandro U: Community-based promotional campaign to improve uptake of intermittent preventive antimalarial treatment in pregnancy in Burkina Faso. Am J Trop Med Hyg 2009, 80:460-469.

23. Ouma PO, Van Eijk AM, Hamel MJ, Sikuku E, Odhiambo F, Munguti K, Ayisi JG, Kager PA, Slutsker L: The effect of health care worker training on the use of intermittent preventive treatment for malaria in pregnancy in rural western Kenya. Trop Med Int Health 2007, 12:953-961.

24. NCAPD, MOMS, MOPHS, KNBS, ICF Macro: Kenya Service Provision Assessment Survey 2010. Nairobi, Kenya: National Coordinating Agency for Population and Development, Ministry of Medical Services, Ministry of Public Health and Sanitation, Kenya National Bureau of Statistic, and ICF Macro; 2011:660.

25. MoHSS, ICF Macro: Namibia Health Facility Census 2009. Windhoek, Namibia: Ministry of Health and Social Services and ICF Macro; 2010:600. 
26. NIS, MoH, Macro International Inc: Rwanda Service Provision Assessment Survey 2007. Claverton, MD: National Institute of Statistics, Ministry of Health, and Macro International Inc; 2008:685

27. NBS, Inc. Ml: Tanzania Service Provision Assessment Survey 2006. Dar es Salaam, Tanzania: National Bureau of Statistics and Macro International Inc; 2007:691.

28. MoH, Macro International Inc: Uganda Service Provision Asssessment Survey 2007. Kampala, Uganda: Ministry of Health and Macro International Inc; 2008:710.

29. NCAPD, MoH, CBS, ORC Macro: Kenya Service Provision Assessment Survey 2004. Nairobi, Kenya: National Coordinating Agency for Population and Development, Ministry of Health, Central Bureau of Statistics, and ORC Macro; 2005:490

30. Zou G: A modified poisson regression approach to prospective studies with binary data. Am J Epidemiol 2004, 159:702-706.

31. Zou GY, Donner A: Extension of the modified Poisson regression model to prospective studies with correlated binary data. Stat Methods Med Res 2013, 22:661-670.

32. Yelland LN, Salter AB, Ryan P: Performance of the modified Poisson regression approach for estimating relative risks from clustered prospective data. Am J Epidemiol 2011, 174:984-992.

33. Betensky RA, Talcott JA, Weeks JC: Binary data with two, non-nested sources of clustering: an analysis of physician recommendations for early prostate cancer treatment. Biostatistics 2000, 1:219-230.

34. Solon G, Haider S, Wooldridge J: What are we weighting for? In NBER Working Paper Series. Cambridge, MA: National Bureau of Economic Research; 2013:29.

35. Miettinen OS: Theoretical Epidemiology: Principles of Occurrence Research in Medicine. New York: Wiley; 1985

36. R Development Core Team: R: A Language and Environment for Statistical Computing. Vienna, Austria: R Foundation for Statistical Computing; 2013.

37. Højsgaard S, Halekoh U, Yan J: The R package geepack for generalized estimating equations. J Stat Softw 2006, 15:1-11

38. Danaei G, Rimm EB, Oza S, Kulkarni SC, Murray CJ, Ezzati M: The promise of prevention: the effects of four preventable risk factors on national life expectancy and life expectancy disparities by race and county in the United States. PLOS Med 2010, 7:e1000248.

39. Lincetto O, Mothebesoanne-Anoh S, Gomez P, Munjanja S: Antenatal Care. In Opportunities for Africa's Newborns - Practical Data, Policy and Programmatic Support for Newborn Care in Africa. Edited by Lord D, Wake R, Elder L, Grear K, Antayhua A. Cape Town, South Africa: The Partnership for Maternal, Newborn \& Child Health; 2006:51-62.

40. KNBS, ICF Macro: Kenya Demographic and Health Survey 2008-09. Calverton, MD: Kenya National Bureau of Statistics and ICF Macro; 2010:430.

41. MoHSS, Inc. MI: Namibia Demographic and Health Survey 2006-07. Windhoek, Namibia and Calverton, MD: Ministry of Health and Social Services and Macro International Inc; 2008:428.

42. MoH, NISR, ICF Macro: Rwanda Interim Demographic and Health Survey 2007-08. Calverton, MD: Ministry of Health, National Institute of Statistics of Rwanda, and ICF Macro; 2009:190

43. NBS, ICF Macro: Tanzania Demographic and Health Survey 2010. Dar es Salaam, Tanzania: National Bureau of Statistics (NBS) [Tanzania] and ICF Macro; 2011:451.

44. UBOS, ICF International Inc: Uganda Demographic and Health Survey 2011 Kampala, Uganda: Uganda Bureau of Statistics and ICF International Inc; 2012:315.

45. Mubyazi GM, Bloch P, Byskov J, Magnussen P, Bygbjerg IC, Hansen KS: Supply-related drivers of staff motivation for providing intermittent preventive treatment of malaria during pregnancy in Tanzania: evidence from two rural districts. Malar J 2012, 11:48

46. Mubyazi GM, Bloch P: Psychosocial, behavioural and health system barriers to delivery and uptake of intermittent preventive treatment of malaria in pregnancy in Tanzania - viewpoints of service providers in Mkuranga and Mufindi districts. BMC Health Serv Res 2014, 14:15.

47. Brentlinger PE, Dgedge M, Correia MA, Rojas AJ, Saúte F, Gimbel-Sherr KH, Stubbs BA, Mercer MA, Gloyd S: Intermittent preventive treatment of malaria during pregnancy in central Mozambique. Bull World Health Organ 2007, 85:873-879.

48. Thiam S, Kimotho V, Gatonga P: Why are IPTp coverage targets so elusive in sub-Saharan Africa? a systematic review of health system barriers. Malar J 2013, 12:353.
49. Mubyazi G, Bloch P, Kamugisha M, Kitua A, ljumba J: Intermittent preventive treatment of malaria during pregnancy: a qualitative study of knowledge, attitudes and practices of district health managers, antenatal care staff and pregnant women in Korogwe District, North-Eastern Tanzania. Malar J 2005, 4:31.

50. Smith LA, Jones C, Adjei RO, Antwi GD, Afrah NA, Greenwood B, Chandramohan D, Tagbor $\mathrm{H}$, Webster J: Intermittent screening and treatment versus intermittent preventive treatment of malaria in pregnancy: user acceptability. Malar J 2010, 9:18.

51. Hill J, Dellicour S, Bruce J, Ouma P, Smedley J, Otieno P, Ombock M, Kariuki S, Desai M, Hamel MJ, ter Kuile FO, Webster J: Effectiveness of antenatal clinics to deliver intermittent preventive treatment and insecticide treated nets for the control of malaria in pregnancy in Kenya. PLoS One 2013, 8:e64913.

52. Pell C, Meñaca A, Afrah NA, Manda-Taylor L, Chatio S, Were F, Hodgson A Hamel MJ, Kalilani L, Tagbor H, Pool R: Prevention and management of malaria during pregnancy: findings from a comparative qualitative study in Ghana, Kenya and Malawi. Malar J 2013, 12:427.

53. Onoka CA, Onwujekwe OE, Hanson K, Uzochukwu BS: Sub-optimal delivery of intermittent preventive treatment for malaria in pregnancy in Nigeria: influence of provider factors. Malar J 2012, 11:317.

54. Mubyazi GM, Magnussen P, Byskov J, Bloch P: Feasibility and coverage of implementing intermittent preventive treatment of malaria in pregnant women contacting private or public clinics in Tanzania: experiencebased viewpoints of health managers in Mkuranga and Mufindi districts. BMC Health Serv Res 2013, 13:372.

55. Gross K, Alba S, Schellenberg J, Kessy F, Mayumana I, Obrist B: The combined effect of determinants on coverage of intermittent preventive treatment of malaria during pregnancy in the Kilombero Valley, Tanzania. Malar J 2011, 10:140.

56. MoHSS: National Malaria Policy. Windhoek, Namibia: Ministry of Health and Social Services; 2005:40.

57. MoHSW: National Guidelines for Diagnosis and Treatment of Malaria. In Malaria Control Series 11. Dar es Salaam, Tanzania: National Malaria Control Programme, Ministry of Health and Social Welfare; 2006:105.

58. Kenya MoH: National Guidelines for Diagnosis, Treatment and Prevention of Malaria for Health Workers in Kenya. Nairobi, Kenya: Division of Malaria Control, Ministry of Health; 2006:43.

59. WHO: WHO Expert Consultation on Cotrimoxazole Prophylaxis in HIV Infection. In WHO Technical Report Series WHO/HIV/200601. Geneva, Switzerland: World Health Organization; 2006:35.

60. Landsberger HA: Hawthorne Revisited. : Management and the Worker: It Critics, and Developments in Human Relations in Industry. Ithaca, N.Y.: Cornell University; 1958.

61. Namusoke F, Ntale M, Wahlgren M, Kironde F, Mirembe F: Validity of selfreported use of sulphadoxine-pyrimethamine intermittent presumptive treatment during pregnancy (IPTp): a cross-sectional study. Malar J 2012, 11:310.

62. Bank W: World Bank Policy Note: Enhancing Public Supply Chain Management in Zambia. Washington, DC: World Bank; 2012.

63. Mikkelsen-Lopez I, Shango W, Barrington J, Ziegler R, Smith T, de Savigny D: The challenge to avoid anti-malarial medicine stock-outs in an era of funding partners: the case of Tanzania. Malar J 2014, 13:181.

doi:10.1186/1475-2875-13-440

Cite this article as: Maheu-Giroux and Castro: Factors affecting providers' delivery of intermittent preventive treatment for malaria in pregnancy: a five-country analysis of national service provision assessment surveys. Malaria Journal 2014 13:440 